\title{
Numerical Investigation of Single-phase Fully Developed Heat Transfer and Pressure Loss in Spirally Corrugated Tubes
}

\author{
Jakob Hærvig $^{1} \quad$ Thomas Condra $^{1} \quad$ Kim Sørensen $^{1}$ \\ ${ }^{1}$ Department of Energy Technology, Aalborg University, 9220-Aalborg, Denmark, \{jah, tc, kso $\}$ @et.aau.dk
}

\begin{abstract}
A numerical study is carried out to investigate heat transfer and friction argumentation in spirally corrugated tubes. 28 geometrically different tubes are investigated to cover a large set of different corrugation characteristics. The pipes investigated have pitch lengths $l / D$ in the range 0.5 to 2.0 and pitch heights $h / D$ in the range 0 to 0.16 . Furthermore, the flow conditions defined by the Reynolds number are investigated for $\operatorname{Re}=5000$ and $R e=10000$, resulting in a total of 56 Computational Fluid Dynamics (CFD) simulations. The performance of the heat exchangers are evaluated based on the Nusselt number, friction factor, and performance evaluation criterion coefficient combining the two into a single unique dimensionless parameter.

The results suggest corrugation to be an effective way to increase the performance of tube heat exchangers. If only heat transfer is considered without paying attention to pressure loss, the optimal tubes have high corrugation heights, where the Nusselt number can be increased by a factor of 2 compared to the non-corrugated tube. If the performance evaluation criterion is used, the optimal geometry has a moderate corrugation height $h / D$ between 0.05 and 0.10 and a low corrugation length $l / D$ around 1.0 , which can be explained by a high increase in pressure loss due to severe corrugation.

Keywords: Stream-wise periodic boundaries, Heat transfer, Pressure loss, 3D CFD simulations, Parameter variation, Fully-developed flow
\end{abstract}

\section{Introduction}

Transferring heat through a straight tube is used in various applications. These applications include but are not limited to power generation, air-conditioning, petrochemical, and diary applications. Two distinct different techniques for enhancing heat transfer are commonly used; namely a passive or active, where the active requires additional power input whereas the passive does not. Therefore, the passive technique is commonly used where the geometry is altered in a more of less sophisticated manner deforming the thermal boundary layer, creating recirculating local flow structures, or larger secondary flow structures flow- ing perpendicular to the main flow. All these phenomena affect both the heat transfer and friction characteristics.

To enhance the forced convection inside a passive heat exchanger tube, two different methods are typically used. One method is to alter the flow by changing the inner geometry of the tube. Another method is to insert loose or fastened geometrical inserts filling the cross-section of the tube, thereby promoting mixing resulting in enhanced heat transfer. These techniques do in general increase the pressure loss as well, which results in the best geometry having a optimal combination of increased heat transfer at slightly higher pressure loss. As a result, numerous studies have already been carried out to investigate the effect of both spirally and transversally tube corrugation.

Even though all studies conclude that heat transfer and pressure drop is increased by corrugation, the literature overview by Vicente et al. (2004) points out that large disagreements between different studies exist. Ganeshan and Rao (1982) investigated seven different spirally corrugated tubes having different width and height of corrugation while suggesting the ratio between heat exchanger capacity to pumping power to be 100 to $150 \%$ more efficient for $\operatorname{Pr}=4.3$ and $\operatorname{Pr}=109$ respectively. As a result, this study suggests the spirally corrugated tubes to be attractive especially for fairly viscous fluids with high Prandtl numbers. Zimparov et al. (1991) conducted experiments on 25 spirally corrugated tubes having pitch height $h / D$ in the range 0.017 to 0.046 and pitch length in the range 0.25 to 0.65 . The study found heat transfer enhancement factors ranging from 1.77 to 2.73 while the friction factor was increased from 100 to $400 \%$. The literature overview by Kareem et al. (2015) gives a great overview of all the studies published in the period 1977 to 2015 . The study clearly shows that the number of publications on passive heat transfer enhancement has increased in recent years, which is attributed an increased awareness of energy savings. Furthermore, the study concludes that even though quite a number of studies already have been published, more parameters should be investigated to cover a larger design space. Therefore, the purpose of this study is to set up a numerical modelling framework that is able to predict both heat transfer and pressure loss in spirally corrugated tubes. Based on this validated framework, a parameter 
variation can be carried out to investigate how different kinds of corrugation affect the performance in terms of heat transfer and pressure loss.

\section{Geometry and Parameters of Inter- est}

\subsection{Terminology and Representation of the Geometry}

The geometry of interest in this study can be described by the following two parameters:

- Corrugation length $l$ : the corrugation length being the stream-wise distance between two successive points where the geometry repeats itself.

- Corrugation height $h$ : the corrugation height being the constant distance between the surface of corrugated tube and the non-corrugated tube with same diameter.

Based on these two parameters, two dimensionless quantities are formed, namely $\Pi_{1}$ and $\Pi_{2}$ :

$$
\begin{aligned}
& \Pi_{1}=l / D, \\
& \Pi_{2}=h / D .
\end{aligned}
$$

The pipe geometry investigated in this study has a constant diameter $D$ and a centre line position which for each repeatable twisted pipe section is fully described by the following parametric trigonometric function for $t \in[0 ; 1]$ :

$$
\begin{aligned}
& x(t)=h \cdot \sin (2 \pi t), \\
& y(t)=h \cdot \cos (2 \pi t), \\
& z(t)=l \cdot t .
\end{aligned}
$$

Based on the parametric representation in (3), the geometries will take the form shown in figure 1 .

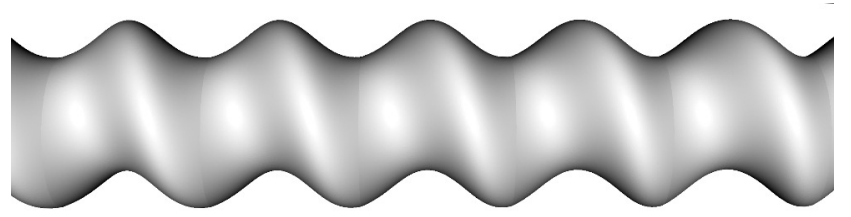

Figure 1. Heat exchanger geometry with $l / D=0.8$ and $h / D=0.2$.

\subsection{Performance Parameters}

To evaluate and compare the performance of the different heat exchangers, two dimensionless quantities describing the heat transfer and pressure loss are used. In the of corrugated tubes, two distinct different phenomena contributed to the pressure loss; wall shear stresses related to the local velocity gradient normal to the tube surface, and pressure drag related to the local pressure distribution along the surface. By integrating the two along the surface and averaging yields a single friction factor parameter that takes both into account. This friction factor is used as a direct measure of the pressure loss in the tube. The Nusselt number and friction factor used to evaluate heat transfer and pressure loss respectively are defined as:

$$
\begin{aligned}
\mathrm{Nu} & =\frac{h D}{k}, \\
f & =\frac{2 \rho \Delta p}{U^{2}} \frac{D}{l} .
\end{aligned}
$$

Instead of evaluating the performance of the corrugated tubes based on two parameters, the performance evaluation criterion $\eta$ is commonly used. This criterion defines the ratio between heat transfer augmentation to friction factor augmentation compared to the non-corrugated straight tube having a Nusselt number $\mathrm{Nu}_{0}$ and friction factor $f_{0}$ :

$$
\eta=\frac{\mathrm{Nu} / \mathrm{Nu}_{0}}{\left(f / f_{0}\right)^{1 / 3}}
$$

That is, if the corrugated tubes have values above 1 , the performance is superior to the non-corrugated tube. To evaluate the performance evaluation criterion, the wellvalidated and commonly used correlations by Gnielinski (1976) and Filonenko (1960) are used:

$$
\begin{aligned}
\mathrm{Nu}_{0} & =\frac{\left(f_{0} / 8\right)(\mathrm{Re}-1000) \operatorname{Pr}}{1+12.7 \sqrt{\left(f_{0} / 8\right)}\left(\operatorname{Pr}^{\frac{2}{3}}-1\right)}\left[1+\left(\frac{D}{L}\right)^{\frac{2}{3}}\right] K, \\
f_{0} & =\left(1.8 \log _{10} \mathrm{Re}-1.5\right)^{-2},
\end{aligned}
$$

where the ratio $D / L$ equals 0 in this study due to the stream-wise periodicity introduced by the boundary conditions described in the following section. The correction factor $K$ used for this study is $\left(T_{\mathrm{b}} / T_{\mathrm{w}}\right)^{n}$ with $n$ being 0.45 for air as discussed by Hufschmidt and Burck (1968) and Yakovlev (1960). In this study, air is used for all simuations. Therefore, the $K$ correction term with the exponent 0.45 is used for all simulations in this study. Should simulations be carried out for different fluids, this exponent should be changed accordingly. 


\section{Numerical Modelling Framework}

\subsection{Governing equations}

The basic equations governing the problem are the continuity (9), momentum (10), and energy (11) equations:

$$
\begin{gathered}
\frac{\partial \rho}{\partial t}+\frac{\partial\left(\rho u_{\mathrm{i}}\right)}{\partial x_{\mathrm{i}}}=0 \\
\frac{\partial\left(\rho u_{\mathrm{i}}\right)}{\partial t}+\frac{\partial\left(\rho u_{\mathrm{i}} u_{\mathrm{j}}\right)}{\partial x_{\mathrm{j}}}=-\frac{\partial p}{\partial x_{\mathrm{i}}}+\frac{\partial}{\partial x_{\mathrm{j}}}\left(\mu \frac{\partial u_{\mathrm{i}}}{\partial x_{\mathrm{j}}}-\rho \overline{u_{\mathrm{i}} u_{\mathrm{j}}}\right) \\
\frac{\partial\left(\rho c_{\mathrm{p}} T\right)}{\partial t}+\frac{\rho c_{\mathrm{p}} u_{\mathrm{j}} T}{\partial x_{\mathrm{j}}}=\frac{\partial}{\partial x_{\mathrm{j}}}\left(k \frac{\partial T}{\partial x_{\mathrm{j}}}-\rho c_{\mathrm{p}} \overline{T u_{\mathrm{j}}}\right)
\end{gathered}
$$

As seen, the temperature is treated as a passive scalar instead of solving the full energy equation. That is, the transport properties do not vary with temperature but are constant. Using this approach, a fully periodic velocity and temperature profile can be obtained. Furthermore, to close the equations and predict the onset of separation accurately, the transition SST turbulence model combining transition modelling by Menter et al. (2006) and SST modelling by Menter (1992) is used.

\subsection{Stream-wise Periodicity}

As the flow in the majority of the heat exchanger tubes is both hydro-dynamically and thermally fully developed, focus will be on this part of the heat exchanger. Instead of simulating the whole heat exchanger starting with a nondeveloped flow and dealing with how the flow develops, periodic boundaries are utilised to couple the inlet and outlet. Using this approach, the inlet and outlet are two-way coupled and therefore a driving force is required to balance out the pressure loss to keep the fluid flowing. Using this approach, the mass flow is specified and the simulation converges towards the corresponding pressure loss. Likewise, modifications are required to keep the temperature from converging towards to wall temperature. The approach suggested by Patankar et al. (1977) is used to make both the temperature and pressure field repeat itself in a periodic manner.

Assuming a constant wall temperature, the fullydeveloped temperature field is obtained by solving for the scaled temperature field $\theta$ as suggested by Patankar et al. (1977):

$$
\theta(x, y, z)=\frac{T(x, y, z)-T_{\mathrm{w}}}{T_{z}^{*}-T_{\mathrm{w}}}
$$

where $T(x, y, z)$ is the temperature field, $T_{\mathrm{w}}$ is the constant wall temperature, and $T_{z}{ }^{*}$ is a local reference temperature profile at the inlet. The reference temperature $T_{z}{ }^{*}$ is given by:

$$
T_{z}^{*}=T_{\mathrm{w}} \frac{\int_{A}\left(T-T_{\mathrm{w}}\right)|\rho \vec{u} d \vec{A}|}{\int_{A}|\rho \vec{u} d \vec{A}|} .
$$

That is, solving equation (12) yields the stream-wise periodic temperature profile, which is used directly to evaluate the local heat transfer coefficient and consequently the local and average Nusselt numbers.

\subsection{Mesh Topology}

A quarter of the cross-sectional mesh is shown in figure 2. The cross-sectional mesh is extruded along the path described by (3) resulting in a structured mesh having the same cross-sectional mesh along the length of the tube. To ensure the results reported in this study are reliable,

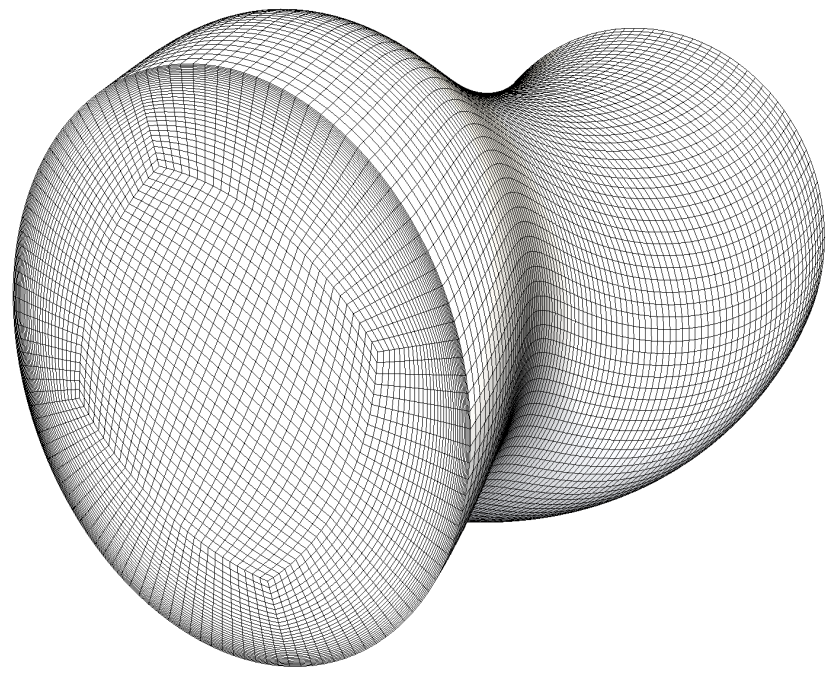

Figure 2. Overview of the structured computational mesh topology used for simulations

two measures are taken. First the number of cells are doubled for a corrugated tube until the results do not change anymore. Instead of simply doubling the number of cells without paying attention how the boundary layer is resolved, the first cell height is kept constant at $y^{+} \approx 1$ for the transition SST turbulence model. This is done for the transition SST model to accurately be able to predict flow seperation at higher corrugations.

\subsection{Data Processing}

As the solution converges, the periodic velocity and temperature profiles are obtained. For the converged solution, the bulk inlet temperature is constant at the specificed value $T_{\text {bulk,in. With the wall temperature held fixed at }}$ a specified value $T_{\text {wall }}<T_{\text {bulk,in }}$, the outlet bulk approaches a temperature between the inlet and wall temperature due to the periodic boundaries. With converged inlet and outlet temperatures, the average convective heat transfer coefficient $h$ and consequently the Nusselt number $\mathrm{Nu}$ are calculated. To get dimensionless friction factor defined by (5), the pressure difference across the domain is calculated. The static pressure is fixed in a cell, and the pressure difference between inlet and outlet is evaluated based on the converged relative pressure field. 


\section{Validation of Results}

Figure 3 shows how the number of cells affects the average Nusselt number. Based on figure 3, the mesh hav-

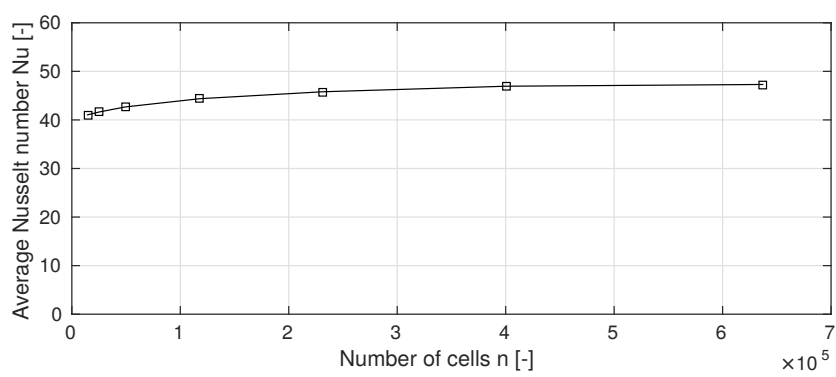

Figure 3. Grid independence study to investigate the importance of cell count for a corrugated pipe $\left(\Pi_{1}=1\right.$ and $\left.\Pi_{2}=0.1\right)$.

ing 231,500 cells is chosen. Using this mesh, the results obtained for a straight tube $\left(\Pi_{1}=1, \Pi_{2}=0\right)$ are in figure 4 and 5 compared to equation (7) and (8) by Gnielinski (1976) and Filonenko (1960) for various Reynolds and Prandtl numbers.

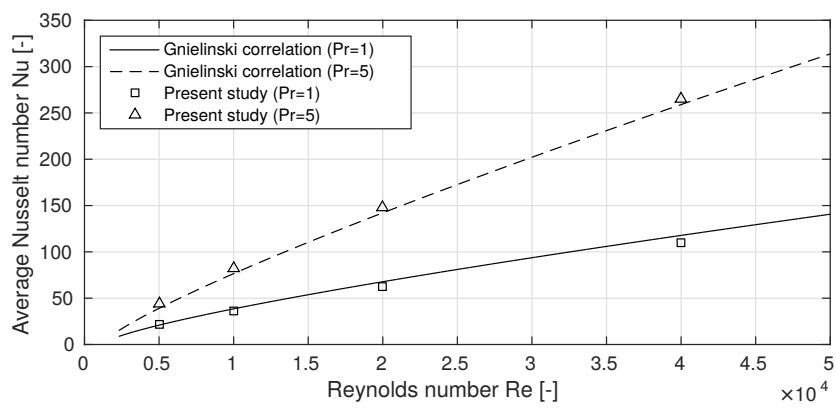

Figure 4. Comparison to the Gnielinski correlation $\left(\Pi_{1}=1\right.$ and $\left.\Pi_{2}=0\right)$.

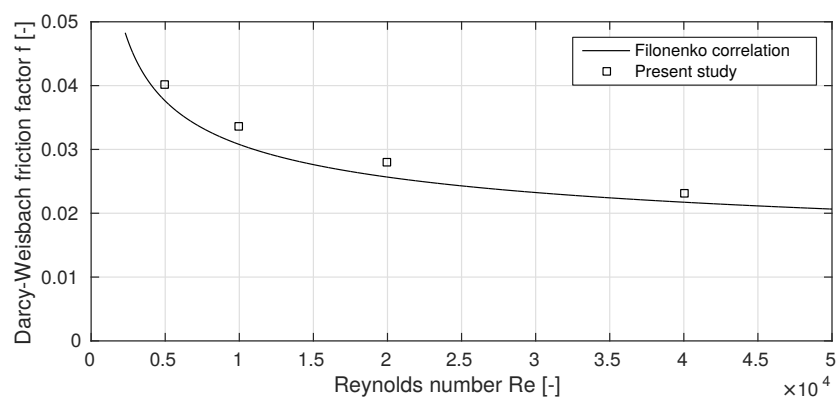

Figure 5. Comparison to the Filonenko correlation $\left(\Pi_{1}=1\right.$ and $\left.\Pi_{2}=0\right)$.

The comparison suggests the CFD simulations to be able to predict smooth pipe performance. For the results in figure 4 and 5 , the Nusselt numbers and friction factors numbers are within a maximum deviation of $6.9 \%$ and $9.4 \%$ respectively.

\section{Results}

In this study, results are reported for corrugations heights $h / D=[0.01,0.03,0.05,0.07,0.10,0.13,0.16]$, corrugations lengths $l / D=[0.5,1.0,1.5,2.0]$ at Reynold numbers $\mathrm{Re}=[5000,10000,20000]$. The performance is evaluated based on the criteria defined in (4), (5), and (6).

\subsection{Heat Transfer Characteristics}

Figure 6 shows contours of constant Nusselt number as function of corrugation height and length at a Reynolds number of 5000. The results in the figure suggests the

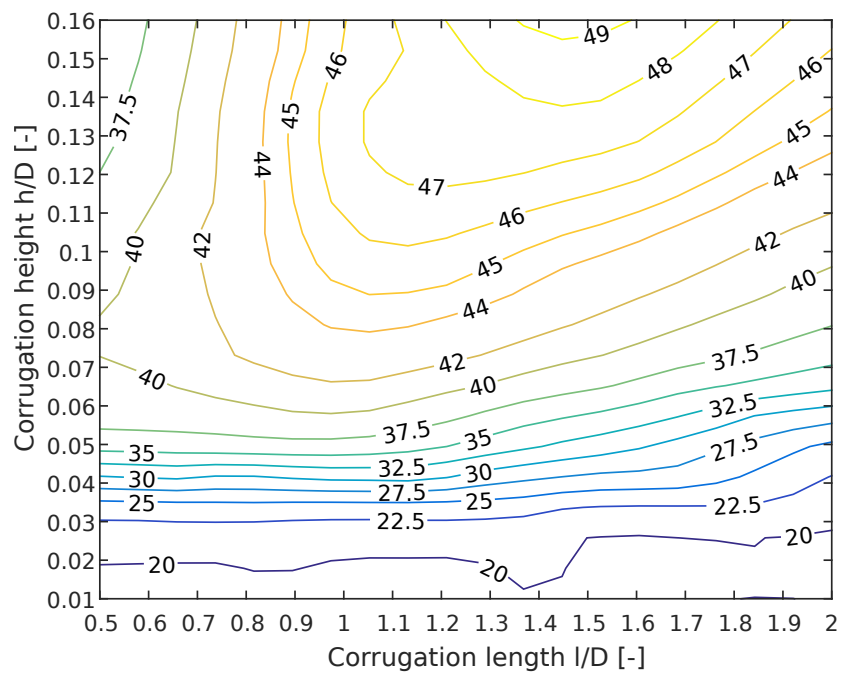

Figure 6. Contours showing the Nusselt number as function of dimensionless corrugation length $l / D$ and height $h / D$ at $\operatorname{Re}=5000$.

Nusselt number to be almost independent of corrugation length in the range $[0.5 ; 2.0]$ at small corrugation heights. This can be explained by the fact that the corrugated tubes approaches the same non-corrugated tube as $h / L$ approaches 0 . At higher corrugation heights, the Nusselt number depends on the corrugation length. At small corrugation lengths $l / D \approx 0.5$, the maximum Nusselt number is obtained at corrugation heights in the range [0.07;0.10]. The decrease in Nusselt number at higher corrugation heights can be explained by the flow being mostly axial and unaffected by the corrugation while the surface area increases. At higher corrugation lengths, the maximum Nusselt number is obtained at higher corrugation heights. Figure 7 shows the results in terms of Nusselt number for $\mathrm{Re}=10000$. In general, higher Nusselt numbers are obtained as a result of higher Reynolds number. At low corrugation heights, the Nusselt number is again almost independent of the corrugation length, increasing slightly with corrugation length. 


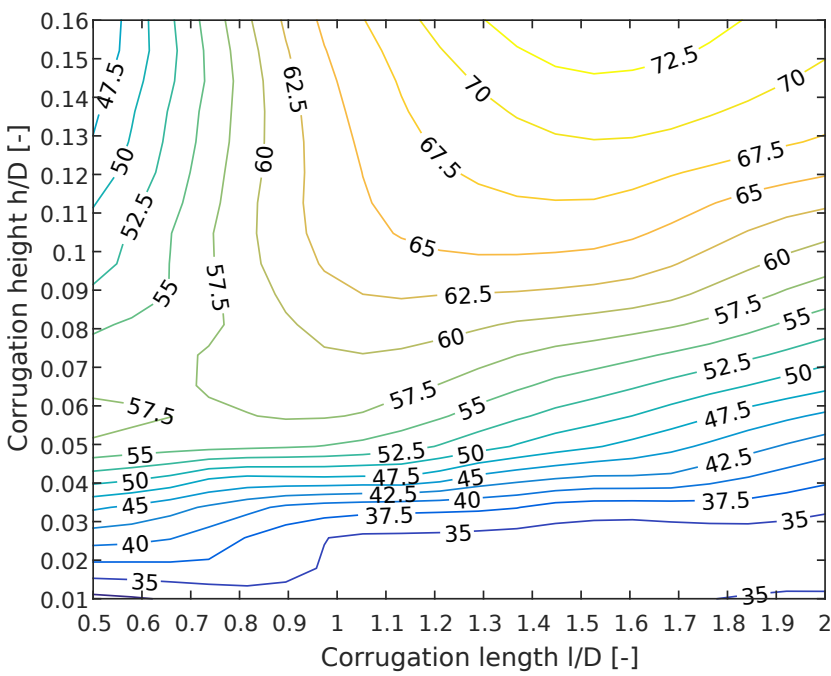

Figure 7. Contours showing the Nusselt number as function of dimensionless corrugation length $l / D$ and height $h / D$ at $\operatorname{Re}=10000$

\subsection{Friction Factor Characteristics}

Figure 8 and 9 shows contours of the friction factor as function of corrugation height and length at a Reynolds numbers of 5000 and 10000 respectively. The figures

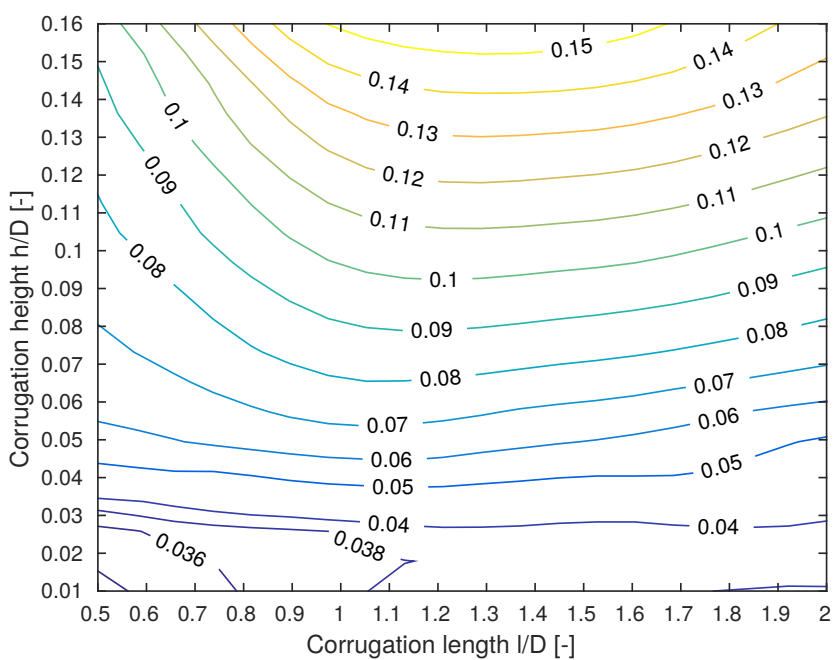

Figure 8. Contours showing the Darcy-Weisbach friction factor as function of dimensionless corrugation length $l / D$ and height $h / D$ at $\operatorname{Re}=5000$.

shows that the friction factor in general increases with corrugation height starting at values close to the noncorrugated pipe at $h / D=0.01$. Furthermore, the results suggest that there is a corrugation length $l / D$ resulting in the highest friction coefficient. This value seems to be almost independent of the Reynolds number at a value between $l / D=1$ and $l / D=1.5$.

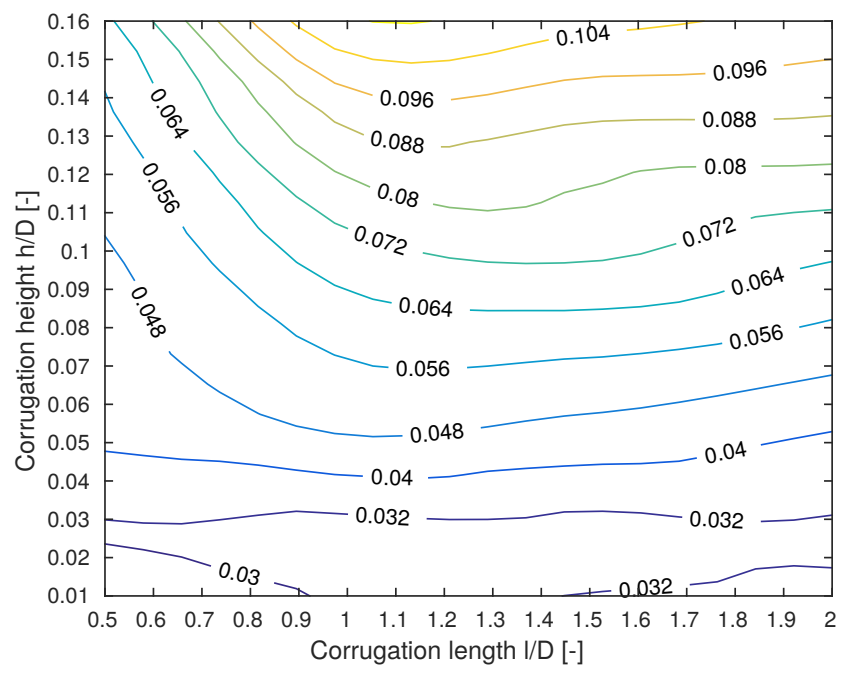

Figure 9. Contours showing the Darcy-Weisbach friction factor as function of dimensionless corrugation length $l / D$ and height $h / D$ at $\operatorname{Re}=10000$.

\subsection{Optimal Combination of Heat Transfer and Pressure loss}

To evaluate the optimal combination of heat transfer and pressure loss, the performance evaluation criterion defined by (6) is used. Figure 10 shows the criterion for the different corrugated tubes at $\operatorname{Re}=5000$. As the results in

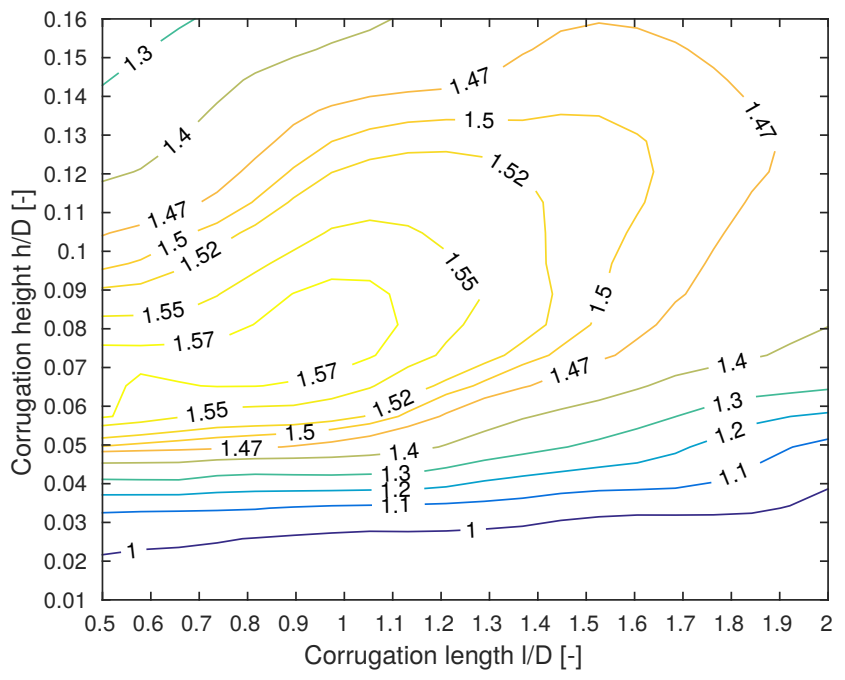

Figure 10. Contours showing the performance parameter $\eta=\left(\mathrm{Nu} / \mathrm{Nu}_{0}\right) /\left(\mathrm{f} / \mathrm{f}_{0}\right)^{1 / 3}$ as function of dimensionless corrugation length $l / D$ and height $h / D$ at $\operatorname{Re}=5000$.

the figure suggest, the optimal combination of heat transfer and pressure loss is found at moderate corrugations heights opposed to when only heat transfer is considered. That is, to obtain the highest Nusselt number, a friction factor is obtained as well. The figure suggests lower corrugations lengths $l / D$ in the range $[0.5 ; 1.0]$ and corrugation heights $h / D$ in the range $[0.05 ; 0.10]$ to be optimal. In this region the performance evaluation criterion is 1.5 . The results for a higher Reynolds number of 10000 are shown in 
figure 11. The results for $\operatorname{Re}=10000$ suggest the max-

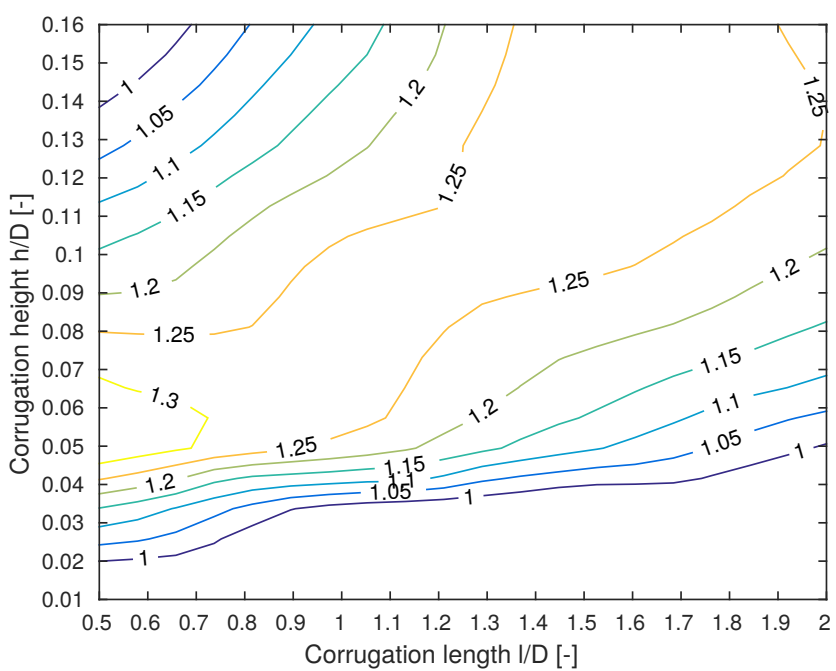

Figure 11. Contours showing the performance parameter $\eta=\left(\mathrm{Nu} / \mathrm{Nu}_{0}\right) /\left(\mathrm{f} / \mathrm{f}_{0}\right)^{1 / 3}$ as function of dimensionless corrugation length $l / D$ and height $h / D$ at $\operatorname{Re}=10000$.

imum performance evaluation criterion to be lower then for $\operatorname{Re}=5000$. At $\operatorname{Re}=10000$, the maximum performance evaluation criterion is again found for corrugated tubes having low corrugation lengths and moderate corrugation heights. For $\mathrm{Re}=10000$, a high criterion can, however, be maintained at higher corrugation heights if the corrugation length is increased accordingly.

\section{Discussion and Conclusion}

A CFD modelling framework to predict heat transfer and pressure loss in spirally corrugated tubes have been presented. The following sums up the findings of this study: (1) In general, both heat transfer and pressure loss are increased when corrugated is introduced. Without paying attention to additional pressure loss, the optimal corrugation is found at highly corrugated tubes having dimensionless corrugation heights $h / D$ and lengths $l / D$ at around $h / D=0.16$ and $l / D=1.5$ for both $\operatorname{Re}=5000$ and $\mathrm{Re}=10000$.

(2) The pressure loss does in general increase with corrugation height $h / D$. The smallest increase is found at low corrugation lengths of $l / D=0.5$. Furthermore, the pressure loss can increase by up to a factor of 4 when introducing a severe corrugation of $h / D=0.16$.

(3) When considering both heat transfer and pressure loss in the performance evaluation, the optimal corrugation is changed. At Reynolds numbers of 5000, the optimal combination corrugation is found at moderate corrugation heights $(h / D=[0.05 ; 0.10])$ and low corrugation lengths $(l / D=[0.5 ; 1.0])$. At these corrugations, performance can be increased by a factor of 1.57 compared to the noncorrugated tube. At $\mathrm{Re}=10000$ the performance is only increased by a factor of 1.25 suggesting corrugation to be more important for lower Reynolds numbers. Again the optimal corrugation is found by a combination of moderate corrugation height. At $\mathrm{Re}=10000$, the optimal corrugation height does however increase with corrugation length.

\section{Acknowledgement}

This work is sponsored by The Danish Council for Strategic Research and the program:

THERMCYC - Advanced thermodynamic cycles for lowtemperature heat sources (No. 1305-00036B).

\section{References}

G.K. Filonenko. Hydraulischer widerstand von rohrleitungen. Teploenergetika, 1:1098-1099, 1960.

S. Ganeshan and M. Rajo Rao. Studies on thermohydraulics of single- and multi-start spirally corrugated tubes for water and time-independent power law fluids. International Journal of Heat and Mass Transfer, 25:10131022, 1982. URL http://dx.doi.org/10.1016/ 0017-9310(82) $90076-\mathrm{X}$.

V. Gnielinski. New equations for heat and mass transfer in turbulent pipe and channel flow. Int. Chem. Eng, 16: 359-368, 1976. URL http: //dx.doi.org/10.1007/ BF 02559682 .

W. Hufschmidt and E. Burck. Der einfluss temperaturabhängiger stoffwerte auf den wärmeübergang bei turbulenter strömung von flüssigkeiten in rohren bei hohen wärmestromdichten und prandtlzahlen. Int. J. Heat Mass Transfer, 11:10411104, 1968. URL http://dx.doi.org/10.1016/ 0017-9310 (68) 90009-4.

Zaid S. Kareem, M. N. Mohd Jaafar, Tholudin M. Lazim, Shahrir Abdullah, and Ammar F. Abdulwahid. Passive heat transfer enhancement review in corrugation. Experimental Thermal and Fluid Science, 68:22-38, 2015. URL http://dx.doi.org/10.1016/j. expthermflusci.2015.04.012.

F. R. Menter. Improved two-equation k-omega turbulence models for aerodynamic flows. Technical report, National Aeronautics and Space Administration, 1992.

F. R. Menter, R. Langtry, and S. Völker. Transition modelling for general purpose cfd codes. Flow Turbulence Combust, 77: 277-303, 2006. URL http: / / dx . doi .org/10 . 1007 / s10494-006-9047-1.

S. V. Patankar, C. H. Liu, and E. M. Sparrow. Fully developed flow and heat transfer in ducts having streamwise-periodic variations of cross-sectional area. Journal of Heat Transfer, 99:180-186, 1977. URL http: //dx . doi .org/10. $1115 / 1.3450666$.

P. G. Vicente, A. García, and A. Viedma. Experimental investigation on heat transfer and frictional characteristics of spirally corrugated tubes in turbulent flow at different prandtl 
numbers. International Journal of Heat and Mass Transfer, 47:671-681, 2004. URL http: //dx. doi.org/10 . 1016/j.ijheatmasstransfer.2003.08.005.

V.V. Yakovlev. Ortliche und mittlere warmeubetragung bei turbulenter rohrstromung nichtsiedenden wassers und hohen warmebelastungen. Kernenergie, 3:1098-1099, 1960.

V. D. Zimparov, N. L. Vulchanov, and L. B. Delov. Heat transfer and friction characteristics of spirally corrugated tubes for power plant condensers -1 . experimental investigation and performance evaluation. International Journal of Heat and Mass Transfer, 34:2187-2197, 1991. URL http://dx. doi.org/10.1016/0017-9310(91)90045-G. 